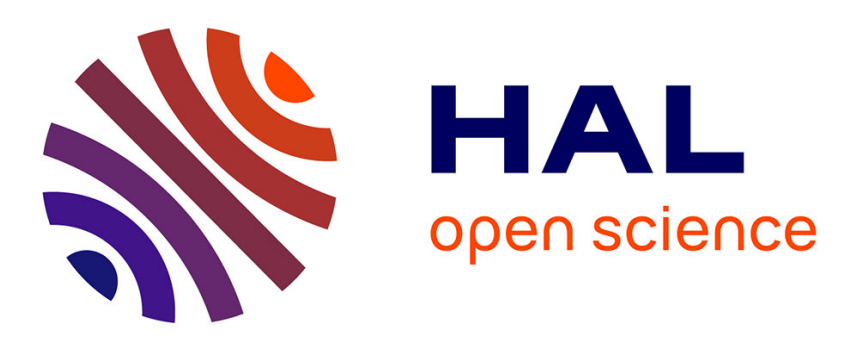

\title{
Aux origines des emplois modaux de l'imparfait. Le cas de l'emploi hypothétique et de l'emploi contrefactuel
}

Adeline Patard, Walter de Mulder

\section{To cite this version:}

Adeline Patard, Walter de Mulder. Aux origines des emplois modaux de l'imparfait. Le cas de l'emploi hypothétique et de l'emploi contrefactuel. Langages, 2014, Syntaxe et sémantique des marqueurs modaux, 1 (193), pp.33-47. 10.3917/lang.193.0033 . hal-01700389

\section{HAL Id: hal-01700389}

\section{https://hal-normandie-univ.archives-ouvertes.fr/hal-01700389}

Submitted on 7 Feb 2018

HAL is a multi-disciplinary open access archive for the deposit and dissemination of scientific research documents, whether they are published or not. The documents may come from teaching and research institutions in France or abroad, or from public or private research centers.
L'archive ouverte pluridisciplinaire HAL, est destinée au dépôt et à la diffusion de documents scientifiques de niveau recherche, publiés ou non, émanant des établissements d'enseignement et de recherche français ou étrangers, des laboratoires publics ou privés. 
Adeline Patard

Université d'Anvers (Belgique)

Walter De Mulder

Université d'Anvers (Belgique)

\section{Aux origines des emplois modaux de l'imparfait. Le cas de l'emploi hypothétique et de l'emploi contrefactuel}

\section{INTRODUCTION}

En français moderne, l'imparfait connaît de nombreux usages " modaux » où il ne renvoie pas à une situation qui a eu lieu dans le passé, mais à une situation dont la factualité est remise en question. On compte, parmi ces emplois, le tour " contrefactuel » (aussi appelé emploi d' « imminence contrecarrée ») (1-2) et le tour hypothétique dans les subordonnées en si (3-4) :

(1) Un pas de plus et cette voiture m'écrasait. (Duhamel, Chronique des Pasquier, 1938)

(2) Si je n'étais pas intervenu, ça se finissait au couteau. (Le canard enchaîné, 21.09.2005)

(3) Si un jour tu partais sans retour / les fleurs perdraient leur parfum. (Piaf, Si tu partais, 1947)

(4) Moi si j'étais un homme, je serais capitaine. (Tell, Si j'étais un homme, 1979)

Dans ces tours, l'imparfait donne lieu à des interprétations qui divergent nettement de sa valeur habituelle de passé imperfectif, valeur à laquelle il est associé dans les descriptions ou dans les séquences à l'arrière-plan de narrations. Le sens ici produit se distingue d'abord sur le plan modal. L'imparfait implique en (1-4) une distance épistémique vis-à-vis de la réalité du locuteur : il renvoie ainsi respectivement, dans les tours contrefactuel et hypothétique, à une situation dont la réalisation a été contrariée (1-2) ou qui apparaît comme improbable (3) voire irréelle (4). 
Par ailleurs, l'interprétation de l'imparfait diffère également sur le plan aspectuel et temporel. Aspectuellement, la morphologie imperfective de l'imparfait se trouve « neutralisée » ou « inhibée » (Iatridou 2000 ; Squartini 2001 ; Caudal, Vetters \& Roussarie 2003) dans la mesure où les interprétations perfectives sont parfois autorisées (5), sans qu'il en résulte aucun effet stylistique ${ }^{1}$ :

(5) Si Jean terminait sa thèse, il hériterait de la fortune de son oncle. (Vet, com. pers.)

(6) Un père à son fils qui vient de grimper le Ventoux :

- Plus entraîné, tu le montais [le Ventoux] en même pas deux heures. (oral conversationnel, in Bres, 2009a : 35)

En effet, en (5), le procès terminer sa thèse décrit à l'aide de l'imparfait, doit avoir atteint son terme pour que le procès conséquent exprimé dans l'apodose (hériter de sa fortune) puisse être envisagé. De même, en (6), le procès contrefactuel à l'imparfait monter [le Ventoux] est délimité dans le temps à l'aide d'un circonstant perfectif de type [en $\mathrm{x}$ temps] (en même pas deux heures) qui impose une vision bornée du procès.

Temporellement, la situation n'est pas (nécessairement) inscrite dans l'époque passée. Dans le tour hypothétique, l'interprétation passée n'est jamais possible : la situation est ancrée, en fonction des indications co(n)textuelles, dans l'époque présente (4) ou future (3). Dans le cas du tour contrefactuel, l'inscription dans une époque donnée n'est pas a priori contrainte par l'imparfait ; la situation peut être présente ou future (voir infra (7)), même s'il est vrai que celle-ci est le plus souvent localisée dans le passé $(1,2$ et 6$)$ :

(7) Henri doit partir prochainement au Portugal sans sa maîtresse Paule : il prétexte un besoin de solitude... Mais il est forcé de lui apprendre qu'une autre jeune femme, Nadine, est du voyage :

- Seul ou avec Nadine, ça ne fait pas beaucoup de différence, dit-il avec mauvaise foi : puisque tu n'es pas jalouse d'elle.

- Ça fait toute la différence du monde ! dit-elle d'une voix bouleversée. Seul, j'étais avec toi, nous restions ensemble. Le premier voyage d'après-guerre : tu n'as pas le droit de le faire avec une autre. (Beauvoir, Les Mandarins, 1954, in Bres, 2009a : 49)

Plusieurs hypothèses ont été avancées pour expliquer la « polysémie » de l'imparfait. Il a d'abord été soutenu que les valeurs modales associées à ce temps sont des effets interprétatifs dérivés de sa valeur de base passée et/ou imperfective (voir entre autres Fleischman 1989 ; Gosselin 1999 ; Wilmet 2003 ; Barceló \& Bres 2006 ou Patard 2007). D’autres linguistes ont avancé que l'imparfait aurait

1. En effet, l'imparfait est aussi compatible avec des interprétations perfectives dans son usage dit « narratif ». Néanmoins, comme il a été souligné à plusieurs reprises dans la littérature (cf. par exemple Caudal \& Vetters 2003 ; Bres 2005 ; Patard 2007), l'usage de l'imparfait produit dans ce cas un effet stylistique qui découle justement de la tension entre des éléments perfectifs du contexte (relation textuelle de progression, procès téliques, circonstants perfectifs, etc.) et la nature imperfective de l'imparfait. 
un sens plus abstrait, qu'il exprimerait, en gros, la « non-actualité » de la situation ou sa non-coïncidence avec la situation d'énonciation (voir entres autres Damourette \& Pichon 1911-1936 ; Le Goffic 1986 ; Touratier 1996 ; De Mulder \& Brisard 2007 ou Brisard 2010) ; selon cette idée, les interprétations temporelles ou modales de l'imparfait seraient des élaborations sémantiques de sa valeur de base construites à partir de spécifications contextuelles.

Le présent article s'interroge sur la genèse des interprétations modales décrites supra dans l'histoire du français. En remontant aux sources de ces interprétations, nous espérons apporter un éclairage diachronique sur la question de la valeur de l'imparfait. Notre article s'organise en trois parties. Nous établirons d'abord dans la section 2 que les deux emplois étudiés, le tour hypothétique et le tour contrefactuel, existaient déjà dès l'ancien français. Dans les sections 3 et 4 , nous nous interrogerons sur l'origine de ces deux emplois en latin et nous tenterons de rendre compte des mécanismes à l'œuvre dans leur développement respectif. Enfin, en conclusion, nous émettrons des hypothèses sur la ou les relations qui existe(nt) en synchronie entre la valeur passée et imperfective de l'imparfait et ses interprétations modales dans les tours contrefactuel et hypothétique.

\section{SITUATION EN ANCIEN FRANÇAIS}

\subsection{L'emploi hypothétique}

L'existence du tour hypothétique [si imparfait, conditionnel] dès l'ancien français est connue et documentée depuis longtemps. Selon la thèse officielle (voir entre autres Sechehaye 1906 ; Brunot 1922), les premières attestations datent du XII ${ }^{\mathrm{e}}$ siècle et se trouvent dans le Couronnement de Louis :

(8) Se ore esteie de son père vengiez, molt en sereie balz et joianz et liez. (Anonyme, Couronnement de Louis, XII siècle, in Wagner, 1939 : 260)

'Si maintenant j'étais vengé de son père, j'en serais heureux, joyeux et content.'

Néanmoins, selon une seconde théorie, défendue de façon convaincante par R. L. Wagner (1939 : 302-303), le tour [si imparfait, conditionnel] pourrait être attesté dès le début du $\mathrm{X}^{\mathrm{e}}$ siècle avec l'occurrence présente dans le Fragment de Valenciennes :

(9) E io ne dolreie de tanta millia hominum, si perdut erent. (Anonyme, Sermon sur Jonas, début du $\mathrm{X}^{\mathrm{e}}$ siècle, in Wagner, 1939 : 303)

'Et je ne pleurerais pas autant de milliers d'hommes, s'ils étaient perdus.'

L'imparfait pouvait également s'employer dans la protase de phrases hypothétiques comportant le subjonctif imparfait dans l'apodose : 
(10) Jo quid si lui veneit a grez,

Tost fust de s'amur aseurez. (Rotelande, Protheselaus, XII ${ }^{\mathrm{e}}$ siècle, in Buridant, $2000: 630)$

'Je crois que s'il lui plaisait, il serait (lit. fût) vite certain de son amour'.

Cependant, ce tour hybride du point de vue du mode, avec la subordonnée à l'indicatif et la principale au subjonctif, semble peu fréquent en ancien français : nous n'en n'avons relevé qu'une seule mention (celle de Buridant) parmi les ouvrages consultés. Comme nous le verrons dans la partie 3, le tour [si imparfait, subjonctif] est plus largement attesté dans plusieurs textes en latin très tardif. Les occurrences telles que (10) pourraient donc constituer une survivance d'un tour plus ancien issu de la période gallo-romane.

Notons enfin que le tour [si imparfait de l'indicatif, conditionnel] était en concurrence en français médiéval avec le tour [si imparfait du subjonctif, imparfait du subjonctif] hérité du latin (11) et ce jusqu'au XV siècle (Wagner, 1939 : 344) :

(11) Se tei ploüst, ici ne volisse estra. (Anonyme, Vie de Saint Alexis, $\mathrm{XI}^{\mathrm{e}}$ siècle, in Wagner, 1939 : 295)

'Si tu le voulais (lit. voulusses) bien, je serais (lit. fusse) ailleurs qu'ici'.

Toutefois, selon R. L. Wagner (1939 : 364), le tour hypothétique moderne était déjà « vainqueur » au XII ${ }^{\mathrm{e}}$ siècle puisqu'il s'imposait dans près de $74,5 \%$ des cas.

\subsection{L'emploi contrefactuel}

Selon R. L. Wagner $(1939: 256)$ et A. Henry (1952:397), les premiers témoignages d'un emploi de l'imparfait pour exprimer « une imminence » ou « une réalisation antidatée $»^{2}$ apparaissent au début du XIV ${ }^{\mathrm{e}}$ siècle. Dans ces exemples, l'imparfait s'emploie dans l'apodose de phrases hypothétiques en $s i$, la protase étant alors au subjonctif :

(12) Tuit estoient perdu, se ne fust li cuens d'Anjou [...] qui les ala rescourre et les enmena sauvement. (Joinville, Vie de Saint Louis, debut du XIV siècle, in Henry, 1952 : 397)

'Tous étaient perdus, s'il ne fût le comte d'Anjou [...] qui les alla délivrer et les emmena sains et saufs.'

Cependant, R. L. Wagner relève (op. cit. : 252), en ancien français, l'existence de systèmes hypothétiques relatifs au passé comportant dans l'apodose un temps passé de l'indicatif (imparfait ou passé simple). Ces constructions pourraient bien correspondre à des emplois contrefactuels. Voici un exemple datant du XII ${ }^{\mathrm{e}}$ siècle :

2. Henry (1952) distingue cette construction fondée sur l'imparfait seul de celle où l'imparfait porte sur aller, devoir ou vouloir signifiant alors « un futur prochain » (ex. Sans le secours du ciel, j'allais encore faire quelque brusquerie ; op. cit. : 394). Le terme d' « imminence contrecarrée » ne doit, selon lui, s'appliquer qu'à ce dernier cas (et non au premier cas basé sur l'imparfait). 
Mes ne poeie a vos venir / Ne fors de mun païs esseir / Se vus ne m'eussiez requis. (M. de France, Ywenec, $\mathrm{XII}^{\mathrm{e}}$ siècle, in Wagner, 1939 : 252)

'Je n'aurais pu (lit. pouvais) venir à vous, ni même sortir de mon pays, si vous ne $\mathrm{m}^{\prime}$ aviez requis.'

Ici, l'imparfait renvoie à une situation passée qui n'a pas été le cas, à savoir l'impossibilité de rejoindre le destinataire et de sortir du pays. Cette situation est bien contrefactuelle, puisque le locuteur a finalement pu faire le déplacement en question. La protase au subjonctif plus-que-parfait se vus ne m'eussiez requis indique alors les circonstances hypothétiques grâce auxquelles la situation décrite ne fut finalement pas le cas.

Il est notable que le tour médiéval diffère sensiblement du tour moderne, entre autres parce que ce dernier s'emploie aussi en dehors de constructions hypothétiques avec si (voir Bres (2007, 2009a) ou Berthonneau \& Kleiber (2003, 2006) pour une description détaillée). Néanmoins, étant donné que le sens produit est exactement le même et que la structure de la construction est similaire (on a affaire dans les deux cas à une structure bipartite), on peut penser qu'il $\mathrm{s}^{\prime}$ agit du même tour, mais que les contraintes linguistiques qui le caractérisent ont évolué depuis l'ancien français.

En conclusion, les tours hypothétique et contrefactuel de l'imparfait existent depuis l'ancienne langue où ils correspondent respectivement à l'emploi de ce temps dans l'un des deux segments d'une phrase hypothétique en si : dans le tour hypothétique, l'imparfait est employé dans la protase alors qu'il apparaît dans l'apodose dans le tour contrefactuel. La position de l'imparfait dans la phrase hypothétique est corrélée à une différence d'interprétation temporelle et épistémique qui permet de distinguer les deux usages que l'on rappelle :

- employé dans la protase, l'imparfait ancre la situation dans le présent ou le futur et signale que celle-ci a une faible chance de se réaliser (mais sa réalisation reste possible),

- employé dans l'apodose, l'imparfait inscrit normalement la situation dans l'époque passée ${ }^{3}$ et la réalisation de celle-ci est posée comme impossible.

Dans les deux parties suivantes, nous tentons de remonter aux sources latines de ces deux emplois de l'imparfait dans l'apodose et dans la protase et de rendre compte de l'émergence des deux interprétations modales qui y sont associées.

3. Nous n'avons trouvé aucune occurrence en français médiéval impliquant une référence non passée. Toutefois, cela n'exclut pas que cette possibilité existait dès l'ancienne langue. 


\section{DÉVELOPPEMENT DE L'IMPARFAIT DANS L'APODOSE}

\subsection{L'expression de l'irréel du passé}

Dès le latin archaïque, on trouve des constructions conditionnelles « mixtes ", dans lesquelles le subjonctif et l'indicatif peuvent s'employer alternativement soit dans l'apodose, soit dans la protase. Il semble toutefois que la combinaison la plus fréquente ait été celle qui comportait l'indicatif dans la principale et le subjonctif dans la subordonnée. L'indicatif soulignait alors la réalité de la situation désignée :

(14) Decem si ad cenam vocasset-PQP.SBJ summos viros nimium opsonavit-PRF.IND. (Plaute, Mercator, 694-695, vers 200 AEC, in Sabanééva, 1996 : 51)

'Même s'il avait invité au souper dix personnages importants, il a acheté trop de choses.'

En latin classique, les structures conditionnelles comportant l'indicatif dans l'apodose se développent et se diversifient. C'est à cette époque que l'emploi de formes indicatives passées s'étend à des conditionnelles hypothétiques exprimant l'irréel du passé. On trouve, de ce fait, un nombre croissant d'imparfaits (15) ou de parfaits (16) employés dans l'apodose de phrases hypothétiques :

Sed is fieri nullo modo poterat-IMP.IND, si Herodotus quidam adessetIMP.SBJ. (Cicéron, Verrines, II.2.128, I $^{\text {er }}$ siècle AEC, in Haverling, 2010 : 144) 'Mais il n'aurait pas pu (lit. ne pouvait pas) avoir cette fonction (celle de grand sacrificateur) si un certain Hérodote avait été présent.'

[...] deleri totus exercitus potuit-PRF.IND, si fugientes persecuti uictores essentIMP.SBJ. (Tite-Live, Histoire de Rome depuis sa fondation, 32.12.7, $\mathrm{I}^{\mathrm{er}}$ siècle AEC, in Haverling, 2010 : 144)

'toute l'armée aurait pu être détruite, si les vainqueurs avaient poursuivi l'ennemi en fuite.'

Ce changement peut s'expliquer par le fait que la forme du subjonctif imparfait, qui s'employait au départ pour exprimer l'irréel dans le passé, était devenue temporellement ambiguë : elle pouvait exprimer l'irréel du passé, mais aussi l'éventualité au présent (Thomas 1938 ; Sabanééva 1996). Dans les hypothétiques référant à des situations révolues, l'emploi de l'imparfait de l'indicatif (cf. poterat en (15)) aurait alors permis de suppléer l'incertitude temporelle qu'aurait produite l'emploi du subjonctif imparfait. Des formes indicatives normalement attachées au domaine du realis auraient donc été " attirées » dans des contextes d'irrealis pour répondre à un besoin expressif : la nécessité d'inscrire la situation envisagée dans l'époque passée. Il est aussi possible que ces formes aient permis stylistiquement au locuteur d'affirmer la réalisation effective de la situation si les conditions adéquates avaient été remplies, par opposition aux formes subjonctives qui soulignaient plutôt l'irréalité de la situation (cf. Harris, 1986 : 416 ; Bybee, Perkins \& Pagliuca, 1994 : 239 ; Haverling, 2010 : 144).

En admettant ces hypothèses, l'imparfait n'exprimerait pas, dans des exemples tels que (15), l'irréalité de la situation dénotée, mais sa fonction principale serait de localiser celle-ci dans le passé. L'interprétation irréelle 
proviendrait alors d'autres éléments du contexte : la conjonction si qui permet de supposer la réalité de la situation sans l'asserter, l'emploi d'un subjonctif imparfait ou plus-que-parfait dans la protase (cf. adesset en (15)), et éventuellement d'autres éléments modalisateurs tels que l'usage d'un verbe modal dans l'apodose (cf. posse en (15)) ou encore d'adjectifs porteurs d'une valeur évaluative (Sabanééva, 1996 : 116).

Par ailleurs, dans certains contextes, l'imparfait semble contribuer lui-même à l'interprétation d'inférences modales grâce à ce que l'on appelle le " paradoxe imperfectif »: lorsque l'imparfait, d'aspect imperfectif, est combiné avec un procès télique, l'atteinte de la culmination est remise en question; il s'ensuit (le plus souvent) une lecture " conative » du procès qui est interprété comme n'ayant pas eu lieu, mais comme ayant été sur le point d'avoir lieu. C'est la lecture que l'on peut faire de l'exemple suivant où l'imparfait se combine avec le verbe venir :

[...] si per Metellum licitum esset-IMP.SB], matres illorum miserorum sororesque ueniebant-IMP.IND [...]. (Cicéron, Verrines, II.5.129, $\mathrm{I}^{\mathrm{er}}$ siècle AEC, in Haverling, $2010:$ 145)

'si Métellus l'avait permis, les mères et les sœurs de ces infortunés seraient venues (lit. venaient).'

L'aspect imperfectif de l'imparfait veniebant permet d'indiquer que le procès n'a pas atteint sa borne culminante, et donc que la venue des mères et des sœurs n'a pas eu lieu.

Les contextes que nous avons décrits supra, où la valeur passée et imperfective de l'imparfait côtoie des effets de sens modaux provenant du contexte (et parfois produits en partie par l'imparfait lui-même), pourraient correspondre à ce que B. Heine (2002) appelle des " bridging contexts », i.e. des contextes transitionnels qui permettent le passage $\mathrm{d}$ 'un sens source à un sens cible, i.e. le changement sémantique d'une forme grammaticale. Pour B. Heine, les « bridging contexts » constituent une première étape vers ce changement sémantique et se manifestent par le fait que le sens source et le sens cible sont contigus dans un contexte donné (le sens source permettant parfois l'inférence du sens cible). C'est bien ce que l'on observe dans le cas présent : le sens passé et imperfectif de l'imparfait côtoie un sens modal d'irréalité porté par le contexte (et éventuellement impliqué par l'usage même de l'imparfait). Ce type de contextes mettant en jeu différents effets de sens adjacents ne donne pas forcément lieu à un changement sémantique subséquent, mais il semble bien que ce mouvement se soit poursuivi avec l'imparfait dans les hypothétiques exprimant l'irréel du passé. C'est ce dont nous allons traiter dans le paragraphe suivant. 


\subsection{De l'irréel du passé au contrefactuel}

En latin classique, l'imparfait a, semble-t-il, eu assez rapidement la possibilité de s'étendre à des hypothétiques dont la référence n'était pas nécessairement passée. Cela est illustré dans l'exemple suivant de Sénèque, où l'imparfait eras renvoie à une situation concomitante avec le moment de la parole :
Si hoc ei optares-IMP.SBJ, cuius nullum beneficium haberes, inhumanum erat-IMP.IND uotum. (Sénèque, Des Bienfaits, 6.26.2, $\mathrm{I}^{\mathrm{er}}$ siècle, in Haverling, $2010: 143)$
'Si tu souhaitais un pareil malheur à celui dont tu n'aurais reçu aucun bienfait, ton vœu serait (lit. était) inhumain.

Du même auteur, on peut citer l'exemple suivant où l'imparfait conserve sa valeur passée (comme dans l'exemple (15) supra) :

$$
\begin{aligned}
& \text { Non poterat-IMP.IND capi nisi capere vellet-IMP.SBJ. (Sénèque, Thyeste, 289- } \\
& 290, \text { I }^{\text {er }} \text { siècle, in Sabanééva, 1996: 116) } \\
& \text { 'Il n'aurait pas pu (lit. ne pouvait pas) être pris, si lui-même n'avait pas } \\
& \text { voulu prendre.' }
\end{aligned}
$$

Ces deux exemples suggèrent que, dans ce type de contexte, l'imparfait de l'indicatif était devenu temporellement ambigu au cours de la période classique, tout comme l'imparfait du subjonctif l'était devenu avant lui.

Sans pouvoir pointer de manière précise la cause de cette extension d'usage de l'imparfait, on peut rappeler que, pendant la période classique, une nouvelle forme récemment constituée et permettant un ancrage passé s'était également propagée dans les hypothétiques exprimant un irréel du passé, pour finalement s'imposer dans une majorité de cas : il s'agit du subjonctif plus-que-parfait (cf. Thomas, 1938 : 219-222 ; Sabanééva, 1996 : 112). Il est possible que l'ambiguïté temporelle de l'imparfait du subjonctif ait laissé le champ libre à différentes formes, dont l'imparfait de l'indicatif et le plus-que-parfait du subjonctif, qui se sont retrouvées, par voie de conséquence, en concurrence pour exprimer le passé dans les hypothétiques. Le plus-que-parfait du subjonctif « ayant remporté la partie » en latin classique, l'extension de l'usage de l'imparfait à des hypothétiques non passées n'a laissé aucun « vide » sémantique nécessitant d'être comblé.

Il reste alors à expliquer l'extension de l'usage de l'imparfait à des contextes hypothétiques non-passés qui s'éloignent davantage de la valeur de base de ce temps. Or, du moment que l'imparfait est étroitement associé à l'idée d'irréalité dans les hypothétiques, rien n'empêche que ce sens irréel se présente seul dans de nouveaux contextes hypothétiques qui s'écartent ainsi du sens de base de l'imparfait. B. Heine (2002) désigne par le terme de " switch contexts » ce type de contextes transitionnels où le sens cible se trouve focalisé dans l'interprétation, tandis que le sens source se trouve relégué à l'arrière-plan, voire effacé. Dans le cas de l'imparfait, l'effacement progressif du sens source s'est clairement opéré au niveau de sa composante temporelle puisque ce temps a acquis la possibilité de décrire des situations hypothétiques non passées (18). 
Par ailleurs, il est plausible que la valeur imperfective de l'imparfait ait aussi été peu à peu mise à l'arrière-plan tandis que le sens d'irréalité devenait le principal élément focalisé, avec pour conséquence l'affaiblissement de l'idée d'imperfectivité portée par l'imparfait dans ce contexte et la disparition du phénomène de paradoxe imperfectif avec les procès téliques (17).

Avant de clore ce paragraphe, nous voudrions souligner le fait que le sens associé à l'imparfait dans les hypothétiques s'est généralisé au stade que nous venons de décrire. Alors que l'inscription de l'événement se limitait au départ à l'époque passée, elle est maintenant également possible à l'époque présente. Néanmoins, selon G. V. Haverling (2010: 148), ce n'est qu'en latin tardif que les hypothétiques à l'imparfait commencent à référer à l'avenir. Le sens de la construction à l'imparfait en latin classique pouvait donc n'être qu'un sens purement « irréel » ou « contrefactuel », sens que les locuteurs pouvaient trouver peu adéquat pour décrire le futur qui, par essence, n'interdit la réalisation d'aucun événement.

\subsection{Du contrefactuel à l'éventuel et le développement de INFINITIF + habebam}

G. V. Haverling relève (2010:148) que, à partir de 400, les hypothétiques employant l'imparfait dans l'apodose commencent à renvoyer à des événements éventuels qui ne sont plus nécessairement irréels (20) et qui peuvent recevoir une lecture future (21). Selon l'auteur, ce type de contextes se rencontre ensuite communément dans les textes du VI ${ }^{\mathrm{e}}-\mathrm{VII}{ }^{\mathrm{e}}$ siècle (21) :

(20) Tamen tu neque diues neque pauper es. Hoc si agnosceres-IMP.SBJ, felix erasIMP.IND. (Anonyme, Le Quérolus, I.II, vers 400 EC, in Haverling, 2010 : 148) 'D'ailleurs, tu n'es ni riche ni pauvre. Si tu pouvais le reconnaître, tu serais (lit. était) heureux.'

(21) Ecce! Pater tuus senuit [...]. Si [...] illi moreretur-IMP.SBJ, recte tibi [...] regnum illius reddebatur-IMP.IND. (Grégoire de Tours, Histoire des Francs, 2.40, $\mathrm{VI}^{\mathrm{e}}$ siècle, in Haverling, 2010 : 148)

'Regarde! Ton père est âgé [...]. Si [...] il venait à mourir, son royaume t'appartiendrait (lit. appartenait) de droit.'

À l'époque où l'on commence à observer l'imparfait dans les hypothétiques exprimant l'éventuel, on note que la périphrase INFINITIF + habebam, l'ancêtre du conditionnel français, se développe également dans ce contexte, ce qui semble tout à fait logique puisque la périphrase est formée à l'aide de l'imparfait :

si diceret-IMP.SBJ " fines terrae », et non diceret " uniuersi fines terrae », dicere habebant-IMP.IND (Augustin d'Hippone, Discours sur les Psaumes, 21.2.28, vers $400 \mathrm{CE}$, in Haverling, 2010 : 150)

's'il disait " les limites de la terre ", et non « toutes les limites de la terre ", ils diraient $\left[\ldots . . .{ }^{\prime}\right.$

Lorsque cette périphrase s'est grammaticalisée entre le latin classique et le latin tardif, celle-ci a pris une valeur de " prédestination » (cf. Benveniste, 1974 : 131 ; 
Bourova, 2002 : 312 ; Thomas 2012). La périphrase indiquait donc que l'événement devait nécessairement avoir lieu :

(23) Nazaraeus vocari habebat-IMP.IND secundum prophetiam (Tertullien, Contre Marcion, 4.8 , III ${ }^{\mathrm{e}}$ siècle)

'Il devait s'appeler le Nazaréen selon la prophétie.'

L'emploi de la périphrase dans les constructions éventuelles a pu être favorisé justement grâce à cette valeur de prédestination. En effet, contrairement aux autres formes concurrentes (les formes subjonctives ou l'imparfait de l'indicatif seul), la périphrase permet de souligner le lien de conséquence inévitable entre la protase et l'apodose. Son développement dans les hypothétiques éventuelles, où elle va peu à peu éliminer toutes les formes concurrentes jusqu'en ancien français, pourrait donc s'expliquer par son sens de prédestination qui vient renforcer expressivement les relations entre les deux membres de la construction hypothétique.

Notons enfin que le tour éventuel avec l'imparfait dans l'apodose (20-21), qui a émergé en bas latin, n'a pas survécu en ancien français, probablement parce qu'il était moins expressif que le tour au conditionnel pour les raisons que nous venons d'invoquer. Cela étant, si le conditionnel a pu s'introduire dans l'apodose de phrases hypothétiques éventuelles, c'est en partie parce que l'imparfait latin avait déjà ouvert la voie avant lui. Néanmoins, comme le signale G. V. Haverling (2010 : 148), l'imparfait semble avoir conservé la possibilité de renvoyer à des situations contrefactuelles en latin tardif. Il se pourrait donc que l'emploi contrefactuel dont nous avons établi l'existence dès l'ancien français pourrait avoir un lien génétique avec les hypothétiques contrefactuelles latines fondées sur l'imparfait.

Après avoir retracé l'émergence de l'imparfait dans l'apodose (section 3.1), tenté d'expliquer l'origine de son interprétation contrefactuelle (section 3.2) et mis en relation ces évolutions avec le développement de la périphrase INFINITIF + habebam dans le même contexte (section 3.3), nous allons maintenant essayer de rendre compte de la genèse de l'emploi hypothétique de l'imparfait dans la protase.

\section{DÉVELOPPEMENT DE L'IMPARFAIT DANS LA PROTASE}

\subsection{De l'interprétation passée à la distanciation épistémique}

Tout comme c'était le cas pour les apodoses, on trouve dès le latin archaïque des exemples dans lesquels la protase comporte un verbe à l'imparfait de l'indicatif. Ce temps sert alors, dans la plupart des cas, à souligner la nature factuelle de la situation désignée, comme dans les exemples suivants : 
- Quid faceret? - Si amabat-IMP.IND, rogas quid faceret ? adseueraret-IMP.SBJ dies nocteisque, in custodia esset semper. (Plaute, Rudens, 378-380, III ${ }^{\mathrm{e}}$ siècle AEC, in Haverling, 2010 : 143)

'- Qu'est-ce qu'il pouvait faire ? - Tu demandes ce qu'il pouvait faire s'il l'aimait ? - Il aurait dû surveiller jour et nuit, monter continuellement la garde.'

Cet emploi est intéressant car l'imparfait y côtoie des effets modaux. Ainsi, en (24), le locuteur semble prendre ses distances avec la réalité de la situation passée amabat assertée par son interlocuteur. Cette interprétation est confirmée par l'apodose au subjonctif qui indique ce que « il » aurait dû faire, s'il avait été vraiment amoureux, à savoir il aurait dû monter la garde jour et nuit. On notera que ce type d'exemple distingue clairement le contenu présupposé de la protase qui n'est pas pris en charge par le locuteur, des informations nouvelles que ce dernier apporte dans l'apodose. Ainsi, ces tours s'apparentent à une forme de discours indirect grâce auquel le locuteur reprend un dire précédent ou présupposé pour le mettre en doute ${ }^{4}$.

L'étude de M. Biraud et S. Mellet (2000) sur le discours indirect en latin est à ce niveau très éclairante. Selon les auteurs, l'imparfait latin permet de souligner, en discours indirect passé, la présence d'un locuteur/énonciateur antérieur, par opposition au subjonctif et à l'infinitif qui impliqueraient une plus grande prise en charge du discours rapporté par le locuteur. L'imparfait permet donc de donner plus de voix à l'énonciateur passé dont on rapporte les propos, en explicitant la prise en charge passée de l'énoncé rapporté. Suivant cette observation, on peut voir dans l'imparfait amabat (24) la marque d'une prise en charge passée de l'énoncé : le locuteur renvoie à un dire antérieur dont il signale l'ancrage passé pour mieux prendre ses distances avec son contenu. En effet, en renvoyant à une énonciation passée, le locuteur ne prend pas en charge l'énoncé au présent et met en doute la réalité de la situation énoncée.

Si l'on accepte cette analyse, la valeur temporelle de l'imparfait reçoit ici une double interprétation : (i) elle inscrit d'abord la situation dénotée dans l'époque passée ; (ii) elle réfère dans le même temps à une énonciation antérieure dont la validité du contenu est mise en doute ; cette dernière interprétation de la valeur passée de l'imparfait est donc à l'origine d'un effet de sens modal épistémique. Nous sommes donc ici en présence de ce que B. Heine (2002) appelle un « bridging context » : le sens (source) de l'imparfait, signifiant une localisation passée et un point de vue imperfectif, y côtoient un sens modal (cible) : la mise en doute de la situation. Cette ambivalence de l'imparfait dans ce type de contexte va être le point de départ d'un nouveau changement sémantique.

4. On pourrait ici parler de « dialogisme » à la suite de Bres (2009b). 


\subsection{Affaiblissement de la valeur passée et imperfective}

Une première étape de l'évolution peut s'observer en latin classique, lorsque l'on trouve des emplois de l'imparfait dans la protase pour décrire des événements téliques, ce qu'illustre l'exemple (25) :
[...] si Caesaris causa in provinciam veniebatis-IMP.IND, ad eum profecto [...] venissetis-PQP.IND. Venistis ad Pompeium. (Cicéron, Pro Q. Ligario, VIII, $\mathrm{I}^{\mathrm{er}}$ siècle, in Sabanééva, $\left.1996: 118\right)$
'[...] si vous étiez venus dans la province à cause de César, vous vous seriez rendus auprès de lui. Vous vous êtes rendus auprès de Pompée.'

Comme pour l'exemple (24), la protase à l'imparfait renvoie à un fait passé et réel, mais la suite de l'exemple (venistis ad Pompeium) montre que la situation désignée ne s'est quand même pas réalisée (ils sont venus, mais pas pour voir César). Ici, le procès à l'imparfait veniebatis ("veniez ») est télique et reçoit une interprétation qui est clairement perfective, l'événement s'est déroulé jusqu'à son terme : les interlocuteurs se sont bien rendus en province. Cet exemple suggère donc que la valeur aspectuelle de l'imparfait s'est affaiblie ; elle serait ici « neutralisée » ou « inhibée » (cf. Iatridou 2000 ; Squartini 2001 ; Caudal, Vetters \& Roussarie 2003) permettant ainsi des interprétations perfectives sans pour autant produire d'effet «narratif ».

On observe plus tardivement, au $\mathrm{VI}^{\mathrm{e}}$ siècle, l'affaiblissement de la valeur temporelle de l'imparfait. Celui-ci s'emploie alors pour décrire des situations non passées, comme dans l'exemple (26) où l'imparfait réfère même à un événement futur :

Melius mihi est mori quam vivere. Si iubebas-IMP.IND, [...] accederemusIMP.SBJ ad prilium; cognuscebas, cui ex nobis sit utelitas an ignavia. (Anonyme, Chronique de Frédégaire, II, 62, VII ${ }^{\mathrm{e}}$ siècle, in Sabanééva, 1996 : 141)

'[...] Si tu l'ordonnais, nous engagerions un combat; tu verrais lequel de nous est vaillant, lequel est lâche.'

Les deux exemples cités correspondent à l'extension de l'imparfait à de nouveaux contextes hypothétiques qui s'éloignent, sur le plan aspectuel et déictique, de sa valeur de base. Pour cette raison, on peut les considérer comme manifestant l'étape des «switch contexts » de B. Heine : la valeur de base de l'imparfait de passé imperfectif a glissé progressivement à l'arrière-plan de l'interprétation, tandis que la valeur modale de distanciation épistémique est mise en avant.

Cette évolution peut être reliée à la disparition progressive, en latin tardif, de l'imparfait du subjonctif au profit du plus-que-parfait du subjonctif (Thomas, 1938 : 241 ; Sabanééva, 1996 : 138), qui tend de plus en plus à s'employer dans des contextes non passés. Selon M. Sabanééva (op. cit. : 144), l'emploi de l'imparfait a ainsi pu être favorisé par le besoin d'exprimer l'éventualité, parallèlement à l'irréalité dénotée par les constructions au plus-que-parfait du subjonctif. En effet, comme on l'a vu en 4.1, l'imparfait permet de mettre en doute la réalité de la situation, sans pour autant basculer complètement dans l'irréel, sans exclure totalement que la situation puisse se réaliser. L'imparfait apparaît donc désormais 
comme une forme tout à fait adéquate pour fonder des hypothétiques exprimant l'éventualité. Il n'est donc pas étonnant de retrouver ensuite, en ancien français, des constructions hypothétiques éventuelles qui emploient l'imparfait de l'indicatif dans la protase. La construction qui s'est alors imposée et qui perdure en français moderne serait donc le résultat d'une rencontre ou d'une hybridation de deux constructions issues du latin tardif : la construction avec l'imparfait dans la protase et celle employant le conditionnel dans l'apodose.

\section{CONCLUSION}

Les emplois hypothétique et contrefactuel de l'imparfait semblent trouver leur origine dans les constructions hypothétiques en latin archaïque et classique, où ils se développent en relation avec l'érosion sémantique des formes subjonctives (notamment l'imparfait et le plus-que-parfait) et pour répondre à des besoins expressifs (marquage d'une référence passée, expression de l'éventualité). Le développement de ces emplois entraîne une modification du sens premier de l'imparfait : à partir du latin tardif, celui-ci s'emploie dans ces constructions avant tout pour y exprimer de valeurs modales : la non-réalisation de la situation dans les hypothétiques contrefactuelles et la distanciation épistémique dans les hypothétiques éventuelles. L'association croissante de l'imparfait avec ces valeurs modales conduit en creux à l'affaiblissement de son sens temporel et aspectuel dans ces contextes, de telle sorte que l'imparfait peut désormais renvoyer à des situations non passées (19 et 26) et se combiner avec de prédicats téliques (25), à l'image de ce que l'on observe en français moderne (1-7).

Le fait que l'imparfait perde ainsi les composantes passée et imperfective de son sens pourrait signifier que ses emplois contrefactuel et hypothétique ne sont plus reliés, dans l'esprit des locuteurs, à la valeur de base de passé imperfectif, mais qu'ils constituent des emplois autonomes, où les nouveaux sens modaux attachés à l'imparfait (contrefactualité et mise à distance) sont indissociables des constructions dans lesquelles ils sont produits.

\section{Références}

BARCeló G. J. \& Bres J. (2006), Les temps de l'indicatif en français, Paris : Ophrys.

Benveniste É. (1974), Problèmes de linguistique générale II, Paris : Gallimard.

Berthonneau A.-M. \& Kleiber G. (2003), " Un imparfait de plus... et le train déraillait ", Cahiers Chronos 11, 1-24.

BERTHONNEAU A.-M. \& KLEIBER G. (2006), " L'imparfait sur les chemins du contrefactuel ", Travaux de linguistique 53, 7-65.

BiRAUD M. \& MelLet S. (2000), "Les faits d'hétérogénéité énonciative dans les textes grecs et latins de l'Antiquité ", Cahiers Chronos 5, 9-48.

BouRova V. (2002), "À la recherche du <conditionnel latin> : les constructions <Infinitif + forme habere> examinées à partir d'un corpus électronique ", in C. D. Pusch, J. Kabatek \& W. Raible (éds), Romanistische Korpuslinguistik II, Tübingen : Gunter Narr Verlag, 303-316. 
BRES J. (2005), L'imparfait narratif, Paris : CNRS-éditions.

BRES J. (2007), "Encore un peu, et l'imparfait était un mode... L'imparfait et la valeur modale de contrefactualité ", Cahiers de praxématique 47, 149-176.

BRES J. (2009a), "Sans l'imparfait, les vendanges tardives ne rentraient pas dans la jupe rhénane... Sur l'imparfait contrefactuel, pour avancer ", Syntaxe et sémantique 10, 33-50.

BRES J. (2009b), " Dialogisme et temps verbaux de l'indicatif ", Langue française 163, 21-39.

BRISARD F. (2010), “Aspects of virtuality in the meaning of the French imparfait”, Linguistics 48 (2), 487-524.

BRUNOT F. (1922), La pensée et la langue, Paris : Masson.

BURIDANT C. (2000), Grammaire nouvelle de l'ancien français, Paris : SEDES.

Bybee J., Perkins R. \& Pagliuca W. (1994), The Evolution of Grammar. Tense, Aspect, and Modality in the Languages of the World, Chicago/London: The University of Chicago Press.

Caudal P. \& VetTers C. (2003), "Un point de vue elliptique sur l'imparfait narratif ", in J. Guéron \& L. Tasmowski (éds), Temps et point de vue, Nanterre : Université Paris X \& Publidix, 103-132.

Caudal P., Vetters C. \& Roussarie L. (2003), " L'imparfait, un temps inconséquent ", Langue française 138, 61-74.

Damourette J. \& Pichon É. (1911-1936 [1970]), Des mots à la pensée. Essai de grammaire de la langue française, vol. 5, Paris : D’Artrey.

De MULDER W. \& BRISARD F. (2007), "L'imparfait marqueur de réalité virtuelle ", Cahiers de praxématique 47, 97-124.

FLeISCHMAN S. (1989), “Temporal distance: A basic linguistic metaphor”, Studies in Language 13, 1-50.

Gosselin L. (1999), "Les valeurs de l'imparfait et du conditionnel dans les systèmes hypothétiques ", Cahiers Chronos 4, 29-51.

HARRIS B. M. (1986), "The historical development of conditional sentences in Romance", Romance Philology 39 (4), 405-436.

HAVERLING G. V. (2010), "On tense, viewpoint and modality from Early to Late Latin", in P. Anreiter \& M. Kienpointner (eds), Latin linguistics today. Akten des 15. internationalen Kolloquiums zur lateinischen Linguistik, Innsbruck : Institut für Sprachen und Literaturen der Universität Innsbruck,143-158.

HEINE B. (2002), "On the role of contexts in grammaticalization", in I. Wischer \& G. Diewald (eds), New Reflections on Grammaticalization, Amsterdam: Benjamins, 83-101.

HenRY A. (1952), " Du subjonctif d'imminence contrecarrée à un passage du Tristan de Béroul ", Romania 73, 392-407.

IATRIDOU S. (2000), “The grammatical ingredients of counterfactuality”, Linguistic Inquiry 31, 231-270.

LE GofFIC P. (1986), "Que l'imparfait n'est pas un temps du passé ", in P. Le Goffic (éd.), Points de vue sur l'imparfait, Caen : Presses Universitaires de Caen, 55-70.

PATARD A. (2007), L'un et le multiple. L'imparfait de l'indicatif en français : valeur en langue et usages en discours, Thèse de l'Université Paul-Valéry - Montpellier 3.

SABANÉÉVA M. (1996), Essai sur l'évolution du subjonctif latin, Louvain : Peeters.

SECHEHAYE A. (1906), "L'imparfait du subjonctif et ses concurrents dans les hypothétiques normales du français ", Romanische Forschungen 19, 341-406.

SQUARTINI M. (2001), “The internal structure of evidentiality in Romance”, Studies in language 25, 297-334. 
THOMAS F. (1938), Recherches sur le subjonctif latin. Histoire et valeur des formes, Paris : Klincksieck.

Thomas J.-F. (2012), "Ultériorité dans le passé et <éventualité> en latin ", Faits de langue 40, 13-20.

TOURATIER C. (1996), Le système verbal français, Paris : Armand Colin.

WAGNER R. L. (1939), Les phrases hypothétiques commençant par si dans la langue française, des origines à la fin du XVle siècle, Paris : Droz.

WILMET M. (2003), Grammaire critique du français, Bruxelles : Duculot. 